\title{
Estimates of Exponential Stability for Solutions of Stochastic Control Systems with Delay
}

\author{
Irada A. Dzhalladova, ${ }^{1}$ Jaromír Baštinec $_{,}{ }^{2}$ Josef Diblík, $^{2,3}$ \\ and Denys Y. Khusainov \\ ${ }^{1}$ Department of Mathematics, Kyiv National Economic University, Peremogy Avenue, Kyiv 03038, Ukraine \\ ${ }^{2}$ Department of Mathematics, Faculty of Electrical Engineering and Communication, Brno University of \\ Technology, Technická 8, 61600 Brno, Czech Republic \\ ${ }^{3}$ Department of Mathematics and Descriptive Geometry, Faculty of Civil Engineering, Brno University of \\ Technology, Veveř́ 331/95, 60200 Brno, Czech Republic \\ ${ }^{4}$ Faculty of Cybernetics, Taras Shevchenko National University of Kyiv, Vladimirskaya Street, \\ Kyiv 01601, Ukraine
}

Correspondence should be addressed to Irada A. Dzhalladova, irada-05@mail.ru

Received 2 February 2011; Accepted 28 March 2011

Academic Editor: Miroslava Růžičková

Copyright (C) 2011 Irada A. Dzhalladova et al. This is an open access article distributed under the Creative Commons Attribution License, which permits unrestricted use, distribution, and reproduction in any medium, provided the original work is properly cited.

\begin{abstract}
A nonlinear stochastic differential-difference control system with delay of neutral type is considered. Sufficient conditions for the exponential stability are derived by using LyapunovKrasovskii functionals of quadratic form with exponential factors. Upper bound estimates for the exponential rate of decay are derived.
\end{abstract}

\section{Introduction}

The theory and applications of functional differential equations form an important part of modern nonlinear dynamics. Such equations are natural mathematical models for various real life phenomena where the aftereffects are intrinsic features of their functioning. In recent years, functional differential equations have been used to model processes in different areas such as population dynamics and ecology, physiology and medicine, economics, and other natural sciences [1-3]. In many of the models the initial data and parameters are subjected to random perturbations, or the dynamical systems themselves represent stochastic processes. For this reason, stochastic functional differential equations are widely studied $[4,5]$.

One of the principal problems of the corresponding mathematical analysis of equations is a comprehensive study of their global dynamics and the related prediction of 
long-term behaviors in applied models. Of course, the problem of stability of a particular solution plays a significant role. Therefore, the study of stability of linear equations is the first natural and important step in the analysis of more complex nonlinear systems.

When applying the mathematical theory to real-world problems a mere statement of the stability in the system is hardly sufficient. In addition to stability as such, it is of significant importance to obtain constructive and verifiable estimates of the rate of convergence of solutions in time. One of the principal tools used in the related studies is the second Lyapunov method [6-8]. For functional differential equations, this method has been developing in two main directions in recent years. The first one is the method of finite Lyapunov functions with the additional assumption of Razumikhin type [9, 10]. The second one is the method of Lyapunov-Krasovskii functionals [11, 12]. For stochastic functional differential equations, some aspects of these two lines of research have been developed, for example, in [11, 13-19] and $[11,18,20-25]$, respectively. In the present paper, by using the method of LyapunovKrasovskii functionals, we derive sufficient conditions for stability together with the rate of convergence to zero of solutions for a class of linear stochastic functional differential equation of a neutral type.

\section{Preliminaries}

In solving control problems for linear systems, very often, a scalar function $u=u(x)$ needs to be found such that the system

$$
\dot{x}(t)=A x(t)+b u(x(t))
$$

is asymptotically stable. Frequently, such a function depends on a scalar argument which is a linear combination of phase coordinates and its graph lies in the first and the third quadrants of the plane. An investigation of the asymptotic stability of systems with a control function

$$
u(x(t))=f(\sigma(t)), \quad \sigma(t)=c^{T} x(t)
$$

that is, an investigation of systems

$$
\dot{x}(t)=A x(t)+b f(\sigma(t)), \quad \sigma(t)=c^{T} x(t),
$$

with a function $f$ satisfying $f(0)=0, f(\sigma)(k \sigma-f(\sigma))>0$ for $\sigma \neq 0$ and a $k>0$ is called an analysis of the absolute stability of control systems [26]. One of the fundamental methods (called a frequency method) was developed by Gelig et al. (see, e.g., the book [27]). Another basic method is the method of Lyapunov's functions and Lyapunov-Krasovskii functionals. Very often, the appropriate Lyapunov functions and Lyapunov-Krasovskii functionals are constructed as quadratic forms with integral terms containing a given nonlinearity [28, 29]. An overview of the present state can be found, for example, in [30, 31]. Problems of absolute stability of stochastic equations are treated, for example, in [11, 14, 15, 24]. 


\section{Main Results}

Consider the following control system of stochastic differential-difference equations of a neutral type

$$
\begin{aligned}
d[x(t)-D x(t-\tau)]= & {\left[A_{0} x(t)+A_{1} x(t-\tau)+a_{2} f(\sigma(t))\right] d t } \\
& +\left[B_{0} x(t)+B_{1} x(t-\tau)+b_{2} f(\sigma(t))\right] d w(t),
\end{aligned}
$$

where

$$
\sigma(t):=c^{T}[x(t)-D x(t-\tau)]
$$

$x:[0, \infty) \rightarrow \mathbb{R}^{n}$ is an $n$-dimensional column vector, $A_{0}, A_{1}, B_{0}, B_{1}$, and $D$ are real $n \times n$ constant matrices, $a_{2}, b_{2}$, and $c$ are $n \times 1$ constant vectors, $f: \mathbb{R} \rightarrow \mathbb{R}$ is a continuous function, $\tau>0$ is a constant delay, and $w(t)$ is a standard scalar Wiener process with

$$
M\{d w(t)\}=0, \quad M\left\{d w^{2}(t)\right\}=d t, \quad M\left\{d w\left(t_{1}\right) d w\left(t_{2}\right), t_{1} \neq t_{2}\right\}=0 .
$$

An $F_{t}$-measurable random process $\{x(t) \equiv x(t, \omega)\}$ is called a solution of (3.1) if it satisfies, with a probability one, the following integral equation

$$
\begin{aligned}
x(t)= & D x(t-\tau)+[x(0)-D x(-\tau)] \\
& +\int_{0}^{t}\left[A_{0} x(s)+A_{1} x(s-\tau)+a_{2} f(\sigma(s))\right] d s \\
& +\int_{0}^{t}\left[B_{0} x(s)+B_{1} x(s-\tau)+b_{2} f(\sigma(s))\right] d w(s), \quad t \geq 0
\end{aligned}
$$

and the initial conditions

$$
x(t)=\varphi(t), \quad x^{\prime}(t)=\psi(t), \quad t \in[-\tau, 0],
$$

where $\varphi, \psi:[-\tau, 0] \rightarrow \mathbb{R}^{n}$ are continuous functions. Here and in the remaining part of the paper, we will assume that the initial functions $\varphi$ and $\psi$ are continuous random processes. Under those assumptions, a solution to the initial value problem (3.1), (3.5) exists and is unique for all $t \geq 0$ up to its stochastic equivalent solution on the space $(\Omega, F, P)[4]$. 
We will use the following norms of matrices and vectors

$$
\begin{aligned}
\|A\| & :=\sqrt{\lambda_{\max }\left(A^{T} A\right)}, \\
\|x(t)\|: & :=\sqrt{\sum_{i=1}^{n} x_{i}^{2}(t),} \\
\|x(t)\|_{\tau} & :=\max _{-\tau \leq s \leq 0}\{\|x(t+s)\|\}, \\
\|x(t)\|_{\tau, \gamma}^{2}: & : \int_{t-\tau}^{t} e^{-\gamma(t-s)}\|x(s)\|^{2} d s,
\end{aligned}
$$

where $\lambda_{\max }(*)$ is the largest eigenvalue of the given symmetric matrix (similarly, the symbol $\lambda_{\min }(*)$ denotes the smallest eigenvalue of the given symmetric matrix), and $\gamma$ is a positive parameter.

Throughout this paper, we assume that the function $f$ satisfies the inequality

$$
0 \leq f(\sigma) \sigma \leq k \sigma^{2}
$$

if $\sigma \in \mathbb{R}$ where $k$ is a positive constant.

For the reader's convenience, we recall that the zero solution of (3.1) is called stable in the square mean if, for every $\varepsilon>0$, there exists a $\delta=\delta(\varepsilon)>0$ such that every solution $x=x(t)$ of (3.1) satisfies $M\left\{\|x(t)\|^{2}\right\}<\varepsilon$ provided that the initial conditions (3.5) are such that $\|\varphi(0)\|_{\tau}<\delta$ and $\|\varphi(0)\|_{\tau}<\delta$. If the zero solution is stable in the square mean and, moreover,

$$
\lim _{t \rightarrow+\infty} M\left\{\|x(t)\|^{2}\right\}=0
$$

then it is called asymptotically stable in the square mean.

Definition 3.1. If there exist positive constants $N, \gamma$, and $\theta$ such that the inequality

$$
M\left\{\|x(t)\|_{\tau, r}^{2}\right\} \leq N\|x(0)\|_{\tau}^{2} e^{-\theta t}
$$

holds on $[0, \infty)$, then the zero solution of (3.1) is called exponentially $\gamma$-integrally stable in the square mean.

In this paper, we prove the exponential $\gamma$-integral stability in the square mean of the differential-difference equation with constant delay (3.1). We employ the method of stochastic Lyapunov-Krasovskii functionals. In [11, 18, 22, 24] the Lyapunov-Krasovskii functional is chosen to be of the form

$$
V[x(t), t]=h[x(t)-c x(t-\tau)]^{2}+g \int_{-\tau}^{0} x^{2}(t+s) d s,
$$


where constants $h>0$ and $g>0$ are such that the total stochastic differential of the functional along solutions is negative definite. form:

In the present paper, we consider the Lyapunov-Krasovskii functional in the following

$$
\begin{aligned}
V[x(t), t]= & {[x(t)-D x(t-\tau)]^{T} H[x(t)-D x(t-\tau)] } \\
& +\int_{t-\tau}^{t} e^{-\gamma(t-s)} x^{T}(s) G x(s) d s+\beta \int_{0}^{\sigma(t)} f(\xi) d \xi,
\end{aligned}
$$

where constants $\gamma>0, \beta>0$ and $n \times n$ positive definite symmetric matrices $G, H$ are to be restricted later on. This allows us not only to derive sufficient conditions for the stability of the zero solution but also to obtain coefficient estimates of the rate of the exponential decay of solutions.

We set

$$
P:=\left(\begin{array}{cc}
H & -H D \\
-D^{T} H & D^{T} H D
\end{array}\right) .
$$

Then, by using introduced norms, the functional (3.11) yields two-sided estimates

$$
\begin{aligned}
\lambda_{\min }(G)\|x(t)\|_{\tau, r}^{2} \leq & V[x(t), t] \leq\left[\lambda_{\max }(P)+0.5 \beta k\|c\|^{2}\right]\|x(t)\|^{2} \\
& +\left[\lambda_{\max }(P)+0.5 \beta k\left\|c^{T} D\right\|^{2}\right]\|x(t-\tau)\|^{2}+\lambda_{\max }(G)\|x(t)\|_{\tau, \gamma^{\prime}}^{2}
\end{aligned}
$$

where $t \in[0, \infty)$.

We will use an auxiliary $(2 n+1) \times(2 n+1)$-dimensional matrix:

$$
S=S(\beta, \gamma, v, G, H):=\left(\begin{array}{lll}
s_{11} & s_{12} & s_{13} \\
s_{21} & s_{22} & s_{23} \\
s_{31} & s_{32} & s_{33}
\end{array}\right)
$$


where

$$
\begin{aligned}
& s_{11}:=-A_{0} H-H A_{0}-B_{0}^{T} H B_{0}-G, \\
& s_{12}:=A_{0}^{T} H D-H A_{1}-B_{0}^{T} H B_{1}, \\
& s_{13}:=-H a_{2}-B_{0}^{T} H b_{2}-\frac{1}{2}\left(\beta A_{0}+v I\right)^{T} c, \\
& s_{21}:=s_{12}^{T}, \\
& s_{22}:=D^{T} H A_{1}+A_{1}^{T} H D-B_{1}^{T} H B_{1}+e^{-\gamma \tau} G, \\
& s_{23}:=D^{T} H a_{2}-B_{1}^{T} H b_{2}-\frac{1}{2} \beta A_{1} c, \\
& s_{31}:=s_{13}^{T} \\
& s_{32}:=s_{23^{\prime}}^{T} \\
& s_{33}:=\frac{v}{k}-b_{2}^{T} H b_{2}-\beta c^{T} a_{2},
\end{aligned}
$$

where $v$ is a parameter.

Now we establish our main result on the exponential $\gamma$-integral stability of a trivial solution in the square mean of system (3.1) when $t \rightarrow \infty$.

Theorem 3.2. Let $\|D\|<1$. Let there exist positive constants $\beta, \gamma, v$ and positive definite symmetric matrices $G, H$ such that the matrix $S$ is positively definite as well. Then the zero solution of the system (3.1) is exponentially $\gamma$-integrally stable in the square mean on $[0, \infty)$. Moreover, every solution $x(t)$ of (3.1) satisfies the inequality

$$
M\left\{\|x(t)\|_{\tau, \gamma}^{2}\right\} \leq N\|x(0)\|_{\tau}^{2} e^{-\theta t}
$$

for all $t \geq 0$ where

$$
\begin{aligned}
N & :=\frac{1}{\lambda_{\min }(G)} \cdot\left(2 \lambda_{\max }(P)+0.5 \beta k\|c\|^{2}+0.5 \beta k\left\|c^{T} D\right\|^{2}+\frac{1}{\gamma} \lambda_{\max }(G)\right), \\
\theta & :=\min \left\{\frac{\gamma \lambda_{\min }(G)}{\lambda_{\max }(G)}, \frac{\lambda_{\min }(S)}{\lambda_{\max }(P)+0.5 \beta k\|c\|^{2}}\right\} .
\end{aligned}
$$


Proof. We will apply the method of Lyapunov-Krasovskii functionals using functional (3.11). Using the Itô formula, we compute the stochastic differential of (3.11) as follows

$$
\begin{aligned}
d V[x(t), t]= & \left(\left[A_{0} x(t)+A_{1} x(t-\tau)+a_{2} f(\sigma(t))\right]^{T} d t\right. \\
& \left.+\left[B_{0} x(t)+B_{1} x(t-\tau)+b_{2} f(\sigma(t))\right]^{T} d w(t)\right) \\
& \times H[x(t)-D x(t-\tau)]+[x(t)-D x(t-\tau)]^{T} \\
& \times H\left(\left[A_{0} x(t)+A_{1} x(t-\tau)+a_{2} f(\sigma(t))\right] d t\right. \\
& \left.\quad+\left[B_{0} x(t)+B_{1} x(t-\tau)+b_{2} f(\sigma(t))\right]^{T} d w(t)\right) \\
+ & {\left[B_{0} x(t)+B_{1} x(t-\tau)+b_{2} f(\sigma(t))\right]^{T} } \\
\times & H\left[B_{0} x(t)+B_{1} x(t-\tau)+b_{2} f(\sigma(t))\right] d\left(w^{2}(t)\right) \\
+ & x^{T}(t) G x(t) d t-e^{-\gamma \tau} x^{T}(t-\tau) G x(t-\tau) d t+\beta f(\sigma(t)) c^{T} \\
\times & \left(\left[A_{0} x(t)+A_{1} x(t-\tau)+a_{2} f(\sigma(t))\right] d t\right. \\
& \left.+\left[B_{0} x(t)+B_{1} x(t-\tau)+b_{2} f(\sigma(t))\right]^{T} d w(t)\right) \\
& -\gamma \int_{t-\tau}^{t} e^{-\gamma(t-s)} x^{T}(s) G x(s) d s d t .
\end{aligned}
$$

Taking the mathematical expectation we obtain (we use properties (3.3))

$$
\begin{aligned}
& M\{d V[x(t), t]\}=M\left\{\left[A_{0} x(t)+A_{1} x(t-\tau)+a_{2} f(\sigma(t))\right]^{T}\right. \\
& \timesH[x(t)-D x(t-\tau)] d t\} \\
&+ M\left\{[x(t)-D x(t-\tau)]^{T}\right. \\
&\left.\times H\left[A_{0} x(t)+A_{1} x(t-\tau)+a_{2} f(\sigma(t))\right] d t\right\} \\
&+ M\left\{\left[B_{0} x(t)+B_{1} x(t-\tau)+b_{2} f(\sigma(t))\right]^{T}\right. \\
&\left.\times H\left[B_{0} x(t)+B_{1} x(t-\tau)+b_{2} f(\sigma(t))\right] d\left(w^{2}(t)\right)\right\} \\
&+ M\left\{\left[x^{T}(t) G x(t) d t-e^{-\gamma \tau} x^{T}(t-\tau) G x(t-\tau) d t\right]\right\} \\
&+ \beta M\left\{f(\sigma(t)) c^{T}\left[A_{0} x(t)+A_{1} x(t-\tau)+a_{2} f(\sigma(t))\right] d t\right\} \\
&-\gamma M\left\{\int_{t-\tau}^{t} e^{-\gamma(t-s)} x^{T}(s) G x(s) d s d t\right\} .
\end{aligned}
$$


Utilizing the matrix $S$ defined by (3.14), the last expression can be rewritten in the following vector matrix form

$$
\begin{aligned}
\frac{d}{d t} M\{V[x(t), t]\}= & -M\left\{\left(x^{T}(t), x^{T}(t-\tau), f(\sigma(t))\right) \times S \times\left(x^{T}(t), x^{T}(t-\tau), f(\sigma(t))\right)^{T}\right\} \\
& -v\left[\sigma(t)-\frac{f(\sigma(t))}{k}\right] f(\sigma(t))-\gamma M\left\{\int_{t-\tau}^{t} e^{-\gamma(t-s)} x^{T}(s) G x(s) d s\right\} .
\end{aligned}
$$

We will show next that solutions of (3.1) decay exponentially by calculating the corresponding exponential rate.

The full derivative of the mathematical expectation for the Lyapunov-Krasovskii functional (3.11) satisfies

$$
\begin{aligned}
\frac{d}{d t} M\{V[x(t), t]\} \leq & -\lambda_{\min }(S) M\left\{\|x(t)\|^{2}\right\} \\
& -\lambda_{\min }(S) M\left\{\|x(t-\tau)\|^{2}\right\} \\
& -\gamma \lambda_{\min }(G) M\left\{\|x(t)\|_{\tau, \gamma}^{2}\right\} .
\end{aligned}
$$

In the following we will use inequalities being a consequence of (3.13).

$$
\begin{aligned}
\lambda_{\min }(G) M\left\{\|x(t)\|_{\tau, r}^{2}\right\} \leq & M\{V[x(t)]\} \\
\leq & {\left[\lambda_{\max }(P)+0.5 \beta k\|c\|^{2}\right] \times M\left\{\|x(t)\|^{2}\right\} } \\
& +\left[\lambda_{\max }(P)+0.5 \beta k\left\|c^{T} D\right\|^{2}\right] M\left\{\|x(t-\tau)\|^{2}\right\} \\
& +\lambda_{\max }(G) M\left\{\|x(t)\|_{\tau, r}^{2}\right\} .
\end{aligned}
$$

Let us derive conditions for the coefficients of (3.1) and parameters of the LyapunovKrasovskii functional (3.11) such that the following inequality:

$$
\frac{d}{d t} M\{V[x(t), t]\} \leq-\theta M\{V[x(t), t]\}
$$

holds. We use a sequence of the following calculations supposing that either inequality

$$
\gamma \lambda_{\min }(G)-\frac{\lambda_{\min }(S)}{\lambda_{\max }(P)+0.5 \beta k|c|^{2}} \lambda_{\max }(G) \geq 0
$$


holds, or the opposite inequality

$$
\gamma \lambda_{\min }(G)-\frac{\lambda_{\min }(S)}{\lambda_{\max }(P)+0.5 \beta k|c|^{2}} \lambda_{\max }(G) \leq 0
$$

is valid. form

(1) Let inequality (3.24) holds. Rewrite the right-hand part of inequality (3.22) in the

$$
\begin{aligned}
-M\left\{\|x(t)\|^{2}\right\} \leq & \frac{1}{\lambda_{\max }(P)+0.5 \beta k\|c\|^{2}} \\
& \times\left[-M\{V[x(t), t]\}+\lambda_{\max }(G) M\left\{\|x(t)\|_{\tau, \gamma}^{2}\right\}\right. \\
& \left.\quad+\left[\lambda_{\max }(P)+0.5 \beta k\left\|c^{T} D\right\|^{2}\right] M\left\{\|x(t-\tau)\|^{2}\right\}\right]
\end{aligned}
$$

and substitute the latter into inequality (3.21). This results in

$$
\begin{aligned}
\frac{d}{d t} M\{V[x(t), t]\} \leq & -\frac{\lambda_{\min }(S)}{\lambda_{\max }(P)+0.5 \beta k\|c\|^{2}} \\
& \times\left[-M\{V[x(t), t]\}+\lambda_{\max }(G) M\left\{\|x(t)\|_{\tau, \gamma}^{2}\right\}\right. \\
& \left.+\left[\lambda_{\max }(P)+0.5 \beta k\left\|c^{T} D\right\|^{2}\right] M\left\{\|x(t-\tau)\|^{2}\right\}\right] \\
& -\gamma \lambda_{\min }(G) M\left\{\|x(t)\|_{\tau, \gamma}^{2}\right\}-\lambda_{\min }(S) M\left\{\|x(t-\tau)\|^{2}\right\},
\end{aligned}
$$

or, equivalently,

$$
\begin{aligned}
\frac{d}{d t} M\{V[x(t), t]\} \leq & -\frac{\lambda_{\min }(S)}{\lambda_{\max }(P)+0.5 \beta k\|c\|^{2}} M\{V[x(t), t]\} \\
& -\lambda_{\min }(S)\left(1-\frac{\lambda_{\max }(P)+0.5 \beta k\left\|c^{T} D\right\|^{2}}{\lambda_{\max }(P)+0.5 \beta k\|c\|^{2}}\right) M\left\{\|x(t-\tau)\|^{2}\right\} \\
& -\left(\gamma \lambda_{\min }(G)-\frac{\lambda_{\min }(S)}{\lambda_{\max }(P)+0.5 \beta k\|c\|^{2}} \lambda_{\max }(G)\right) M\left\{\|x(t)\|_{\tau, r}^{2}\right\}
\end{aligned}
$$

The inequality

$$
\frac{\lambda_{\max }(P)+0.5 \beta k\left\|c^{T} D\right\|^{2}}{\lambda_{\max }(P)+0.5 \beta k\|c\|^{2}} \leq 1
$$


always holds. Because inequality (3.24) is valid, a differential inequality

$$
\begin{aligned}
\frac{d}{d t} M\{V[x(t), t]\} & \leq-\frac{\lambda_{\min }(S)}{\lambda_{\max }(P)+0.5 \beta k\|c\|^{2}} M\{V[x(t), t]\} \\
& \leq-\theta M\{V[x(t), t]\}
\end{aligned}
$$

will be true as well.

(2) Let inequality (3.25) hold. We rewrite the right-hand side of inequality (3.22) in the form

$$
\begin{aligned}
-M\left\{\|x(t)\|_{\tau, \gamma}^{2}\right\} \leq \frac{1}{\lambda_{\max }(G)} \times & \left(-M\{V[x(t), t]\}+\left(\lambda_{\max }(P)+0.5 \beta k\|c\|^{2}\right) M\left\{\|x(t)\|^{2}\right\}\right. \\
& \left.+\left[\lambda_{\max }(P)+0.5 \beta k\left\|c^{T} D\right\|^{2}\right] M\left\{\|x(t-\tau)\|^{2}\right\}\right)
\end{aligned}
$$

and substitute the latter again into inequality (3.21). This results in

$$
\begin{aligned}
\frac{d}{d t} M\{V[x(t), t]\} \leq & -\lambda_{\min }(S) M\left\{\|x(t)\|^{2}\right\}-\lambda_{\min }(S) M\left\{\|x(t-\tau)\|^{2}\right\}+\gamma \frac{\lambda_{\min }(G)}{\lambda_{\max }(G)} \\
\times & \left\{-M\{V[x(t), t]\}+\left(\lambda_{\max }(P)+0.5 \beta k\|c\|^{2}\right) M\left\{\|x(t)\|^{2}\right\}\right. \\
& \left.+\left[\lambda_{\max }(P)+0.5 \beta k\left\|c^{T} D\right\|^{2}\right] M\left\{\|x(t-\tau)\|^{2}\right\}\right\}
\end{aligned}
$$

or in

$$
\begin{aligned}
\frac{d}{d t} M\{V[x(t), t]\} \leq & -\gamma \frac{\lambda_{\min }(G)}{\lambda_{\max }(G)} M\{V[x(t), t]\} \\
& -\left(\lambda_{\min }(S)-\frac{\lambda_{\max }(P)+0.5 \beta k\|c\|^{2}}{\lambda_{\max }(G)} \gamma \lambda_{\min }(G)\right) M\left\{\|x(t)\|^{2}\right\} \\
& -\left(\lambda_{\min }(S)-\frac{\gamma \lambda_{\min }(G)\left[\lambda_{\max }(P)+0.5 \beta k\left\|c^{T} D\right\|^{2}\right]}{\lambda_{\max }(G)}\right) M\left\{\|x(t-\tau)\|^{2}\right\} .
\end{aligned}
$$

Because inequality (3.25) is valid, a differential inequality

$$
\frac{d}{d t} M\{V[x(t), t]\} \leq-\gamma \frac{\lambda_{\min }(G)}{\lambda_{\max }(G)} M\{V[x(t), t]\} \leq-\theta M\{V[x(t), t]\}
$$

will be valid as well. 
Analysing inequalities (3.30) and (3.34) we conclude that (3.23) always holds. Solving inequality (3.23) we obtain

$$
M\{V[x(t), t]\} \leq M\{V[x(0), 0]\} e^{-\theta t} .
$$

Now we derive estimates of the rate of the exponential decay of solutions. We use inequalities (3.22), (3.35). It is easy to see that

$$
\begin{aligned}
\lambda_{\min }(G) M\left\{\|x(t)\|_{\tau, r}^{2}\right\} \leq & M\{V[x(t), t]\} \leq M\{V[x(0), 0]\} e^{-\theta t} \\
\leq & \left(\left(\lambda_{\max }(P)+0.5 \beta k\|c\|^{2}\right)\|x(0)\|^{2}\right. \\
& \left.\quad+\left[\lambda_{\max }(P)+0.5 \beta k\left\|c^{T} D\right\|^{2}\right]\|x(-\tau)\|^{2}+\lambda_{\max }(G)\|x(0)\|_{\tau, \gamma}^{2}\right) e^{-\theta t} \\
\leq & \left(2 \lambda_{\max }(P)+0.5 \beta k\|c\|^{2}+0.5 \beta k\left\|c^{T} D\right\|^{2}+\frac{1}{r} \lambda_{\max }(G)\right)\|x(0)\|_{\tau}^{2} e^{-\theta t} .
\end{aligned}
$$

Now, inequality (3.16) is a simple consequence of the latter chain of inequalities.

\section{A Scalar Case}

As an example, we will apply Theorem 3.2 to a scalar control stochastic differential-difference equation of a neutral type

$$
\begin{aligned}
d\left[x(t)-d_{0} x(t-\tau)\right]= & {\left[a_{0} x(t)+a_{1} x(t-\tau)+a_{2} f(\sigma(t))\right] d t } \\
& +\left[b_{0} x(t)+b_{1} x(t-\tau)+b_{2} f(\sigma(t))\right] d w(t),
\end{aligned}
$$

where $\sigma(t)=c\left[x(t)-d_{0} x(t-\tau)\right], x \in \mathbb{R}, a_{0}, a_{1}, a_{2}, b_{0}, b_{1}, d_{2}, d_{0}$, and $c$ are real constants, $\tau>0$ is a constant delay, and $w(t)$ is a standard scalar Wiener process satisfying (3.3). An $F_{t}$-measurable random process $\{x(t) \equiv x(t, \omega)\}$ is called a solution of (4.1) if it satisfies, with a probability one, the following integral equation:

$$
\begin{aligned}
x(t)= & d_{0} x(t-\tau)+\left[x(0)-d_{0} x(-\tau)\right] \\
& +\int_{0}^{t}\left[a_{0} x(s)+a_{1} x(s-\tau)+a_{2} f(\sigma(t))\right] d s \\
& +\int_{0}^{t}\left[b_{0} x(s)+b_{1} x(s-\tau)+b_{2} f(\sigma(t))\right] d w(s), \quad t \geq 0 .
\end{aligned}
$$


The Lyapunov-Krasovskii functional $V$ reduces to

$$
V[x(t), t]=\left[x(t)-d_{0} x(t-\tau)\right]^{2}+g \int_{t-\tau}^{t} e^{-\gamma(t-s)} x^{2}(s) d s+\beta \int_{0}^{\sigma(t)} f(\xi) d \xi,
$$

where we assume $g>0$ and $\beta>0$. The matrix $S$ reduces to (for simplicity we set $H=(1)$ )

$$
S=S(g, \beta, \gamma, v):=\left(\begin{array}{lll}
s_{11} & s_{12} & s_{13} \\
s_{21} & s_{22} & s_{23} \\
s_{31} & s_{32} & s_{33}
\end{array}\right)
$$

and has entries

$$
\begin{aligned}
& s_{11}:=-2 a_{0}-b_{0}^{2}-g, \\
& s_{12}:=a_{0} d_{0}-a_{1}-b_{0} b_{1}, \\
& s_{13}:=-a_{2}-b_{0} b_{2}-\frac{1}{2}\left(\beta a_{0}+v\right) c, \\
& s_{21}:=s_{12} \\
& s_{22}:=2 a_{1} d_{0}-b_{1}^{2}+e^{-\gamma \tau} g, \\
& s_{23}:=a_{2} d_{0}-b_{1} b_{2}-0.5 \beta a_{1} c, \\
& s_{31}:=s_{13}, \\
& s_{32}:=s_{23}, \\
& s_{33}:=\frac{v}{k}-b_{2}^{2}-\beta c a_{2},
\end{aligned}
$$

where $v$ is a parameter. Therefore, the above calculation yields the following result.

Theorem 4.1. Let $\left|d_{0}\right|<1$. Assume that positive constants $\beta, \gamma, g$, and $v$ are such that the matrix $S$ is positive definite. Then the zero solution of (4.1) is exponentially $\gamma$-integrally stable in the square mean on $[0, \infty)$. Moreover, every solution $x(t)$ satisfies the following convergence estimate:

$$
M\left\{\|x(t)\|_{\tau, \gamma}^{2}\right\} \leq N\|x(0)\|_{\tau}^{2} e^{-\theta t}
$$

for all $t \geq 0$ where

$$
\begin{gathered}
N:=\frac{1}{g}\left(2+2 d_{0}^{2}+0.5 \beta k c^{2}+0.5 \beta k\left(c d_{0}\right)^{2}\right)+\frac{1}{\gamma}, \\
\theta:=\min \left\{r, \frac{\lambda_{\min }(S)}{1+d_{0}^{2}+0.5 \beta k c^{2}}\right\} .
\end{gathered}
$$




\section{Acknowledgments}

The second author was supported by Grant P201/10/1032 of Czech Grant Agency, and by Project FEKT/FSI-S-11-1-1159. The third author was supported by Grant P201/11/0768 of Czech Grant Agency, and by the Council of Czech Government MSM 0021630503. The fourth author was supported by Project M/34-2008 of Ukrainian Ministry of Education, Ukraine and by Grant P201/10/1032 of Czech Grant Agency.

\section{References}

[1] L. Glass and M. C. Mackey, From Clocks to Chaos, The Rhythms of Life, Princeton University Press, Princeton, NJ, USA, 1988.

[2] V. Kolmanovskii and A. Myshkis, Introduction to the Theory and Applications of Functional-Differential Equations, vol. 463 of Mathematics and Its Applications, Kluwer Academic, Dordrecht, The Netheralnds, 1999.

[3] Y. Kuang, Delay Differential Equations with Applications in Population Dynamics, vol. 191 of Mathematics in Science and Engineering, Academic Press, Boston, Mass, USA, 1993.

[4] I. I. Gikhman and A. V. Skorokhod, Stochastic Differential Equations, Naukova Dumka, Kiev, Ukraine, 1968.

[5] R. Z. Khasminskii, Stability of Systems of Differential Equations under Stochastic Perturbations of Parameters, Science Publishing House, Moscow, Russia, 1969.

[6] A. A. Martynuk and A. A. Martynjuk, Stability of Motion of Complex Systems, Naukova Dumka, Kiev, Ukraine, 1975.

[7] E. A. Barbashin, Lyapunov's Functions, Nauka, Moskow, Russia, 1970.

[8] E. A. Barbashin, Introduction to the Theory of Stability, Wolters-Noordhoff, Groningen, The Netherlands, 1970.

[9] D. Ya. Khusainov and A. V. Shatyrko, Method of Lyapunov Functions in the Study of Stability Functional Differential Systems, Publishing Company of Kiev National University, Kiev, Ukraine, 1997.

[10] B. S. Razumikhin, Stability in Hereditary Systems, Science Publishing House, Moscow, Russia, 1988.

[11] D. G. Korenevskii, Stability of Solutions in Deterministic and Stochastic Differential-Difference Equations, Algebraic Criteria, Naukova Dumka, Kiev, Ukraine, 1992.

[12] N. N. Krasovskii, Some Problems in the Stability Theory of Motion, Fiziko-Tekhnicheskikh, Moscow, Russia, 1959.

[13] A. S. Bychkov and D. Khusainov, "An exponential decay of solutions of neutral type stochastic equations," Random Operators and Stochastic Equations, vol. 3, no. 3, pp. 245-256, 1995.

[14] I. A. Dzhalladova, "Investigation of the stabilization of a mathematical model of a dynamical system with random action in the case of resonance," Ukrainian Mathematical Journal, vol. 49, no. 9, pp. 13241329, 1997, translated from Ukrans ki Matematichni Zhurnal, vol. 49, no. 9, pp. 1177-1181.

[15] I. A. Dzhalladova, "Investigation of the stabilization of a mathematical model of a dynamical system with random influence in the resonance case," Ukrainian Mathematical Journal, vol. 50, no. 8, pp. 12991307, 1998, translated from Ukrans ki Matematichni Zhurnal, vol. 50, no. 8, pp. 1137-1143.

[16] D. Ya. Khusainov and A. S. Bychkov, "Stability of stochastic systems of neutral type with small deviations of the argument," Differential Equations, vol. 28, no. 12, pp. 2060-2069, 1992.

[17] F. L. Lewis, Optimal Estimation, With an Introduction to Stochastic Control Theory, Wiley-Interscience Publication, John Wiley \& Sons, New York, NY, USA, 1986.

[18] D. G. Korenevskiǔ, Stability of Dynamical Systems under Random Perturbations of Parameters, Algebraic Criteria, Naukova Dumka, Kiev, Ukraine, 1989.

[19] I. G. Nechaeva and D. Y. Khusainov, "Obtaining estimates for the stability of solutions of stochastic functional-differential equations," Differential Equations, vol. 28, no. 3, p. 405-414, 547, 1992.

[20] J.-H. Park and S. Won, "A note on stability of neutral delay-differential systems," Journal of the Franklin Institute, vol. 336, no. 3, pp. 543-548, 1999.

[21] V. Rezvan, Absolute Stability Automatic System with Delay, Nauka, Moscow, Russia, 1983.

[22] M. L. Sverdan, E. F. Tsarkov, and V. K. Yasinskii, Stability in Stochastic Modeling of Complex Dynamical Systems, Svyatyn, "Nad Prutom", 1996.

[23] E. F. Tsarkov, Random Perturbations of Functional Difefrential Equations, Zinatne, Riga, Latvia, 1989. 
[24] E. F. Tsarkov and V. K. Yasinskii, Quasi-Linear Stochastic Functional Differential Equations, Orientir, Riga, Latvia, 1992.

[25] X.-x. Liu and B. Xu, "A further note on stability criterion of linear neutral delay-differential systems," Journal of the Franklin Institute, vol. 343, no. 6, pp. 630-634, 2006.

[26] M. A. Ajzerman and F. R. Gantmacher, Absolute Stability Regulations System, Science Publishing House, Moscow, Russia, 1963.

[27] A. H. Gelig, G. A. Leonov, and V. A. Jakubovič, The Stability of Nonlinear Systems with a Nonunique Equilibrium State, Nauka, Moscow, Russia, 1978.

[28] A. K. Gelig and G. A. Leonov, Nonlinear Systems, Nauka, Moscow, Russia, 1978.

[29] A. D. Myshkis, Linear Differential Equations with Delayed Argument, Nauka, Moscow, Russia, 2nd edition, 1972.

[30] E. Boukas, Deterministic and Stochastic Time Delay Systems, Birkhäuser, Boston, Mass, USA, 2002.

[31] X. Liao and P. Yu, Absolute Stability of Nonlinear Control Systems, vol. 25 of Mathematical Modelling: Theory and Applications, Springer, New York, NY, USA, 2nd edition, 2008. 


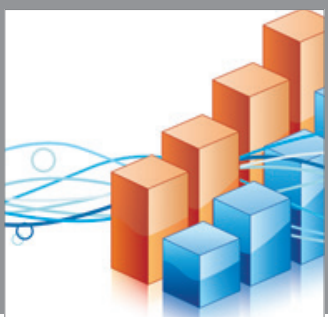

Advances in

Operations Research

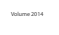

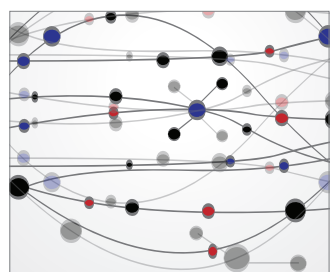

\section{The Scientific} World Journal
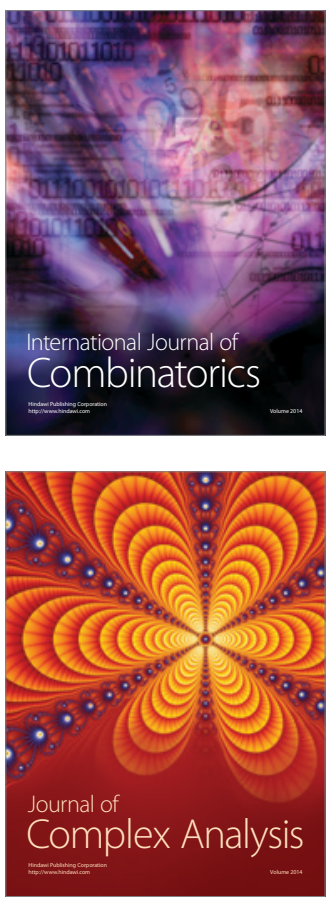

International Journal of

Mathematics and

Mathematical

Sciences
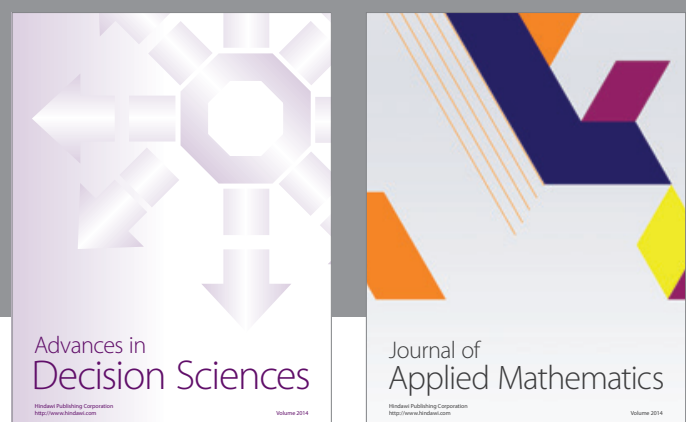

Journal of

Applied Mathematics
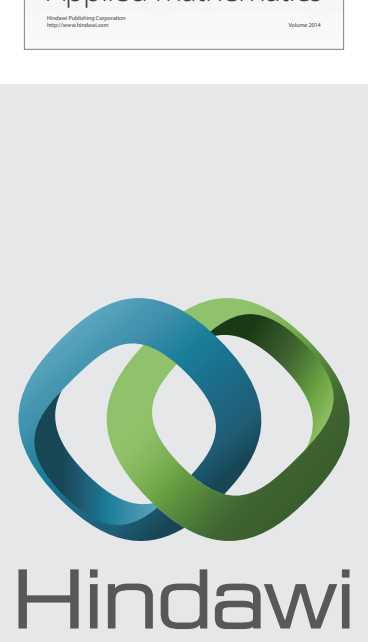

Submit your manuscripts at http://www.hindawi.com
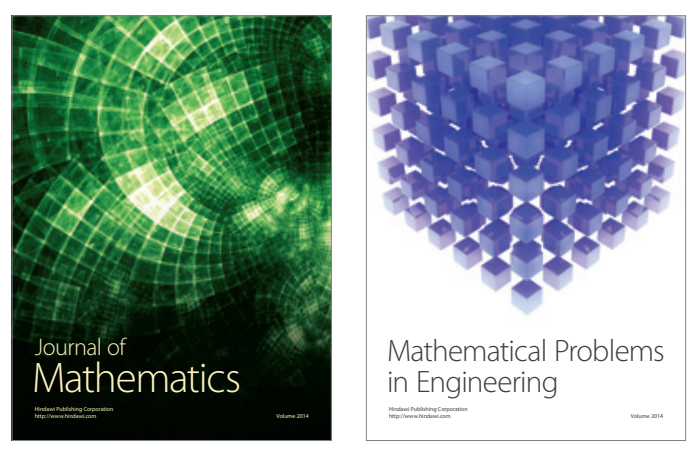

Mathematical Problems in Engineering
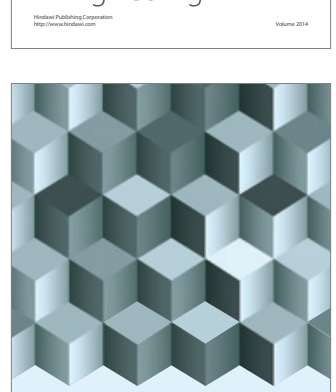

Journal of

Function Spaces
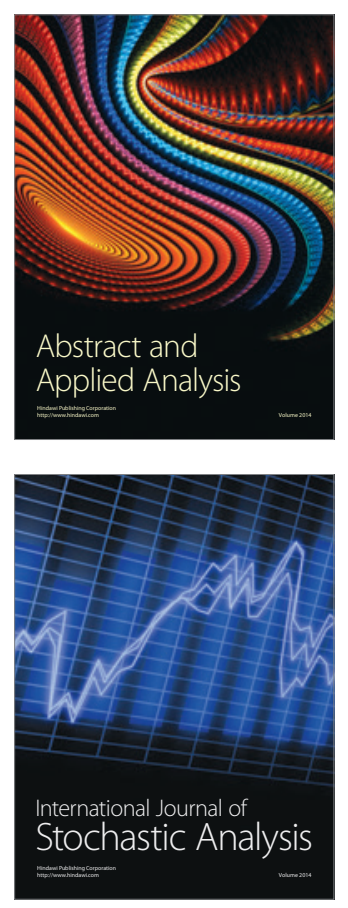

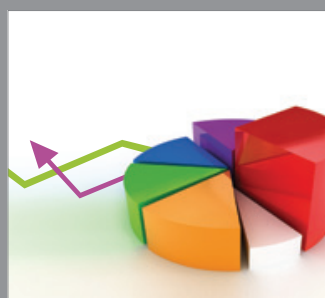

ournal of

Probability and Statistics

Promensencen
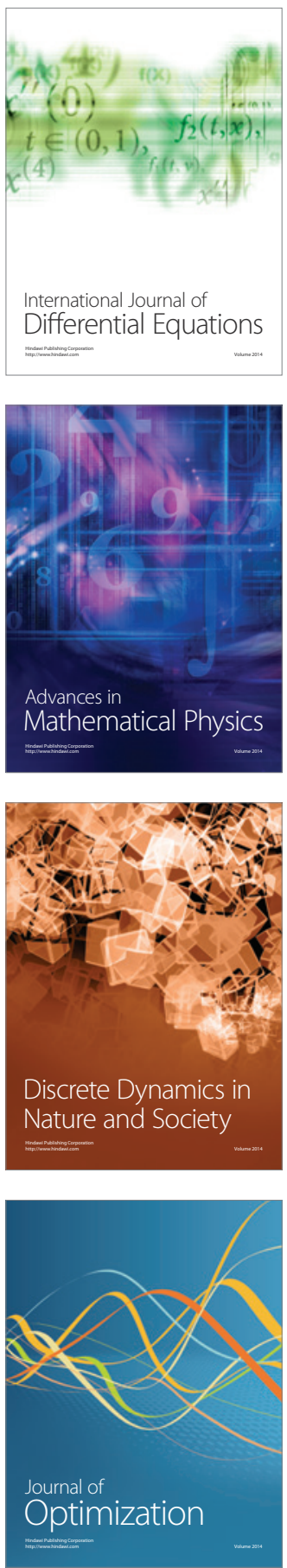